\title{
De explicatione per series trigonometricas instituenda functionum unius variabilis arbitrariarum, et praecipue earum, quae per variabilis spatium finitum valorum maximorum et minimorum numerum habent infinitum, disquisitio.
}

\author{
(Auctore R. Lipschitz.)
}

Series secundum sinus et cosinus multiplorum anguli cujusdam progredientes, quibus functio unius variabilis arbitraria explicetur, per diversas analyseos disciplinas maximo in usu esse inter omnes earum rerum peritos jam pridem constat. Geometrarum igitur munus esse videbatur, ut latum functionum illarum campum perscrutarentur atque limitem agerent inter èas, quae seriebus trigonometricis evolvi possint, atque eas, quae hanc proprietatem non habeant. Quo munere perfunctus est, ut conatus ab aliis antea factos praetereamus, illustrissimus Lejeune-Dirichlet edita commentatione celeberrima, quae inscribitur: „Sur la convergence des séries trigonométriques qui servent à représenter une fonction arbitraire entre des limites données" (Diar. Crell. Vol. IV. pag. 157). Permagna quae inde cepit analysis incrementa leviter adumbrare liceat, ut quaestionum a nobis institutarum et hoc libello pertractatarum quae sit vis et natura dilucidius perspiciatur.

Summi viri commentatio ei superstructa est fundamento, quod data functione arbitraria $\varphi(x)$ pro intervallo variabilis $x$ a valore $-\pi$ usque ad valorem $+\pi$ extenso, et formata serie

$$
\frac{1}{2 \pi} \int_{-\pi}^{+\pi} \varphi(\alpha) d \alpha+\frac{1}{\pi}\left\{\begin{array}{l}
\cos x \int_{-\pi}^{+\pi} \varphi(\alpha) \cos \alpha d \alpha+\cos 2 x \int_{-\pi}^{+\pi} \varphi(\alpha) \cos 2 \alpha d \alpha+\cdots \\
\sin x \int_{-\pi}^{+\pi} \varphi(\alpha) \sin \alpha d \alpha+\sin 2 x \int_{-\pi}^{+\pi} \varphi(\alpha) \sin 2 \alpha d \alpha+\cdots
\end{array}\right.
$$

aggregatum ex $2 n+1$ primis seriei terminis per integrale definitum

$$
\text { (2.) } \frac{1}{\pi} \int_{-\pi}^{+\pi} \varphi(\alpha) \frac{\sin \left(n+\frac{1}{2}\right)(\alpha-x)}{\sin \frac{1}{2}(\alpha-x)} \dot{d} \alpha
$$


exprimitur, et quod, si integrale numero $n$ in infinitum crescente ad certum quendam valorem quam proxime accedat, hic valor nihil est nisi summa seriei (1.) in infinitum protractae. Tota instituitur disquisitio ea conditione, ut functio $\varphi(x)$ per intervallum a $-\pi$ usque ad $+\pi$ extensum sit finita atque finitum numerum solutionum continuitatis et finitum numerum valorum maximorum et minimorum praebeat. Demonstratur autem, quod sequitur,

Theorema I. Quantitatibus $g$ et $h$ explentibus conditiones signis $0 \leqq g<h \leqq \frac{\pi}{2}$ descriptas sit functio $f(\beta)$ a valore $\beta=g$ usque ad valorem $\beta=h$ data semper finita et continua atque aut ita comparata, ut per totum spatium interjectum vel crescat vel immutata maneat, aut ita comparata, ut per totum spatium vel decrescat vel immutata maneat, tum integrale $\int_{g}^{h} f(\beta) \frac{\sin \dot{k} \beta}{\sin \beta} d \beta$ quantitate positiva $k$ omnem modum excedente certo cuidam valori appropinquat. Qui valor, si quantitas $g$ est positiva, cifrae aequalis, si quantitas $g$ est cifrae aequalis, $=\frac{\pi}{2} f(0)$ evadit.

Ex hoc theoremate sub conditionibus supra dictis concluditur, integrale (2.) numero $n$ in infinitum crescente ad valorem $\frac{1}{2}(\varphi(x-\varepsilon)+\varphi(x+\varepsilon))$ convergere (dum signo $\varepsilon$ quantitatem infinite parvam notamus), itaque seriem (1.) esse convergentem, quicunque valor quantitati $x$ assignetur. Leguntur autem in fine commentationis allatae haecce: „Il nous resterait à considérer les cas, où les suppositions que nous avons faites sur le nombre des solutions de continuité et sur celui des valeurs maxima et minima cessent d'avoir lieu. Ces cas singuliers peuvent être ramenés à ceux que nous venons de considérer". Et verbis quibusdam interjectis: "Mais la chose, pour être faite avec toute la clarté qu'on peut désirer exige quelques détails liés aux principes fondamentaux de l'analyse infinitésimale et qui seront exposés dans une autre note, dans laquelle je m'occuperai aussi de quelques autres propriétés assez remarquables de la série (7.)", quae est series nostra (1.). Quam commentationem nunquam in lucem esse prolatam quum geometris valde dolendum sit, disquisitionum illarum vestigia reperire et repertis ingredi, quantum possimus et valeamus, propositum nobis est. Atque primum quae ex scriptis illustrissimi Lejeune-Dirichlet conjungere contigit commemoremus. Habemus enim praeter commentationem supra allatam aliam ejusdem demonstrationis expositionem in repertorio physico a clarissimis Dove et Moser edito, et commentationem famosam, quae inscribitur: „Sur les séries dont le terme général dépend de 
deux angles, et qui servent à exprimer des fonctions arbitraires entre des limites données" (Diar. Crell. Vol. XVII. pag. 35).

Functiones $\varphi(x)$, quae non satisfaciant conditionibus supra expositis, in tria genera distribui possunt, quum intra spatium a $-\pi$ usque ad $+\pi$ extensum aut valores infinite magnos accipiant, aut infinitum numerum solutionum continuitatis, aut infinitum numerum valorum maximorum et minimorum habeant. Quos tres casus per se quemque ut ita dicam in jus vocare sufficiet, quia duobus vel tribus simul adcitis seriei (1.) species potius quam vis et natura mutatur.

Quibus finibus functio $\varphi(x)$ primis duobus casibus arceatur videbis, dum perpendis conditiones, quibus fit, ut integralia definita, quae in serie (1.) coefficientium partes agunt, certam habeant significationem. Primo enim casu, si quantitates quaedam inter $-\pi$ et $+\pi$ positae signis $a$ et $b$ notantur, necesse est integrale $\int_{a}^{b} \varphi(x) d x$ manere functionem finitam et continuam quantitatum $a$ et $\left.b^{*}\right)$. Sint $c_{1}, c_{2}, \ldots c_{\mu}$ singuli quantitatis $x$ valores, quibus respondent valores functionis $x$ infinite magni, sint $\delta_{1}, \delta_{2}, \ldots \delta_{\mu}$ quantitates quamvis exiguae, excidi possunt e spatio a $-\pi$ usque ad $+\pi$ extenso $\mu$ intervalla limitibus

$$
c_{1}-\delta_{1} \text { et } c_{1}+\delta_{1}, \quad c_{2}-\delta \text { et } c_{2}+\delta_{2}, \ldots c_{\mu}-\delta_{\mu} \text { et } e_{\mu}+\delta_{\mu}
$$

inclusa, quae primae speciei intervalla vocamus. Quod vero restat spatium conflatum erit e finito numero intervallorum ad alteram speciem referendorum, per quorum quodque functio $\varphi(x)$ sufficit conditionibus, quae in theoremate $I$. functioni $f(\beta)$ praescriptae sunt. Casu autem altero, quo functio $\varphi(x)$ in finito spatio inter $-\pi$ et $+\pi$ intermisso infinite multas solutiones continuitatis praebet, si quantitates quaedam inter $-\pi$ et $+\pi$ positae signis $a$ et $b$ notantur, necesse est semper fieri posse, ut inter $a$ et $b$ aliae quantitates $r$ et $s$ interserantur, inter quas functio $\varphi(x)$ finita et continua maneat $\left.{ }^{* *}\right)$. Unde rite ratiocinando sequitur, spatium a $-\pi$ usque ad $+\pi$ extensum in finitum numerum intervallorum discerpi posse, quorum binae species binis primi casus speciebus sint similes. Prioris speciei intervallum quodque finitae sed ad libitum díminuendae est magnitudinis et infinitum solutionum continuitatis continet numerum, quorum

*) Diar. Crell. Vol. XVII. pag. 54.

**) Diar. Crell. Vol. IV. pag. 169. 
intervallorum limites aeque ac supra signis

$$
c_{1}-\delta_{1} \text { et } c_{1}+\delta_{1}, \quad c_{2}-\delta_{2} \text { et } c_{2}+\delta_{2}, \ldots c_{\mu}-\delta_{\mu} \text { et } c_{\mu}+\delta^{\mu}
$$

significamus. Alterius speciei intervallum quodque eam habet indolem, at per totum intervallum functio $\varphi(x)$ sufficiat conditionibus, quibus in theoremate I. functio $f(\beta)$ adstricta sit. Nunc vero, si convergentiam seriei (1.) dijudicare velis, rei cardo versatur in quaestione instituenda, num integrale (2.) numero $n$ in infinitum crescente certo cuidam valori appropinquet nec ne. Qua in re si quantitati $x$ valor inter $-\pi$ et $+\pi$ situs assignatur quilibet, dummodo nulli quantitatum $c_{1}, c_{2}, \ldots c_{\mu}$ aequalis sit, quantitatibus exiguis $\delta_{1}, \delta_{2}, \ldots \delta_{\mu}$ commode electis tum primo quum altero casu semper effici potest, ut valor ille quantitatis $x$ cadat in intervallum, quod alterius speciei dicimus. Quum autem integrale (2.) extendatur a valore $\alpha=-\pi$ usque ad valorem $\alpha=+\pi$, discerpatur spatium in modum supra expositum, ut integrale fiat aequale aggregato ex integralibus formae ejusdem, quorum limites sint limites intervallorum vel prioris vel alterius speciei appellatorum. Itaque integralia prioris speciei limitibus inclusa ad priorem integralium speciem, alterius speciei limitibus inclusa ad alteram speciem referimus. Prioris speciei integralia quantitatibus $\delta_{1}, \delta_{2}, \ldots \delta_{\mu}$ apte electis ad quamlibet exiguitatem deprimi posse, quicunque valor numero $n$ tribuatur, casu quidem primo ostendit illustrissimus Lejeune-Dirichlet Diar. Crell. Vol. XVII. pag. 55, casu autem secundo ei innititur fundamento, quod integralium quodcunque est aggregatum ex infinite multis integralibus ita constitutis, ut aggregatum et ipsum quantumvis minuatur. Alterius speciei integralia ita sunt comparata, ut adcito theoremate I. qui sit valor, ad quem quodque in infinitum crescente numero $n$ quam proxime accedat, mox appareat. Unde gignitur, ultra ullum modum crescente numero $n$, valor integralis (2.) $\frac{1}{2}(\varphi(x-\varepsilon)+\varphi(x+\varepsilon)$ ) (ubi signo $\varepsilon$ quantitatem infinite parvam denotamus), qni est summa seriei (1.) in infinitum protractae aequalis; atque patet, sub conditionibus supra dictis seriem nostram esse convergentem pro quovis valore quantitatis $x$ inter $-\pi$ et $+\pi$ posito, exceptis valoribus $c_{1}$, $c_{2}, \ldots c_{\mu}$. Neque vero negandum singulis quibusdam casibus fieri posse, ut series (1.) etiam pro quibusdam horum valorum certam nanciscatur significationem; quorum casuum investigatione hoc loco supersedemus.

Jam restat, ut de casu tertio agamus, quo functio $\varphi(x)$ in intervallo a $-\pi$ usque ad $+\pi$ extenso est continua, finitum tantum numerum solutionum continuitatis sed infinitum numerum habet valorum minimorum et maximorum. Nulla sane in scriptis illustrissimi Lejeune-Dirichlet inveniuntur adjumenta, 
quibus ad quaestiones has enodandas via patefiat, nisi haec verba ad omnes tres casus referenda, quae leguntur in commentationum allatarum prima ${ }^{*}$ ): „La restriction que je viens de préciser, et celle de ne pas devenir infinie, sont les seules auxquelles la fonction $\varphi(x)$ soit sujette et tous les cas qu'elles n'excluent pas peuvent être ramenés à ceux que nous avons considérés dans ce qui précède". Qua in re teneas restrictionem hic commemoratam esse eam, quae ad casum alterum nostrum pertineat, neque casum primum (ubi functioni $\dot{\varphi}(\boldsymbol{x})$ valores infinite magni competant) antea venisse in disquisitionem quam commentatione illa, quae inscripta est: „Sur les séries dont le terme général dépend de deux angles etc." Itaque in casum tertium summa qua possumus diligentia inquiramus.

Discrimen sane magnum est inter casus priores et casum tertium, quum casibus illis functio $\varphi(x)$ adstringatur conditionibus, quibus fit, ut integralia definita, quibus coefficientes seriei (1.) exprimuntur, certam teneant significationem, hoc vero casu conditiones eas expletas esse res ipsa indicet. Si enim $a_{1}, a_{2}, \ldots a_{v}$ sunt valores variabilis $x$, quibus functionis $\varphi(x)$ continuitas deletur, integralium $\int_{-\pi}^{+\pi} \varphi(\alpha) \cos n \alpha d \alpha$ et $\int_{-\pi}^{+\pi} \varphi(\alpha) \sin n \alpha d \alpha$ quodque est aequale aggregato ex integralibus formae ejusdem, quae limitibus $-\pi$ et $a_{1}$, $a_{1}$ et $a_{2}, \ldots a_{v}$ et $\pi$ includuntur, nec dubitari potest, quin singula singulorum aggregatorum integralia ideoque aggregata omnia certam habeant significationem. Nam integralis definiti notio innititur continuitati functionis per intervallum integrationis servatae, neque tollitur, si infinitus numerus valorum maximorum et minimorum in intervallo integrationis finito invenitur. Duobus vero modis - omnino diversis accidere potest, ut per finitum variabilis intervallum infinitus sit numerus valorum maximorum et minimorum functioni competentium.

Intervallum enim si limitibus $a$ et $b$ includitur, aut interjicere potes inter $a$ et $b$ quantitates $r-\delta$ et $r+\delta$ eum in finem, ut valorum maximorum et minimorum functioni $\varphi(x)$ competentium numerus sit finitus inter $a$ et $r-\delta$, finitus inter $r+\delta$ et $b$, infinitus vero inter quantitates $r-\delta$ et $r+\delta$, quantitate positiva finita sed quantumvis deprimenda $2 \delta$ inter se distantes; aut res ita se habet, ut quaecunque quantitates $r$ et $s$ in intervallo a $x=a$ usque ad $x=b$ extenso ponantur finita quantitate inter se distantes, finitus valorum maximorum et minimorum numerus inter $r$ et $s$ numquam inveniri possit; aut intervallum

*) Diar. Crell. Vol. IV. pag. 169. 
datum continet finitum numerum spatiorum finitorum, in quorum quoque aut hoc aut illud eveniat. Brevitatis causa rem ita enuntiemus, ut dicamus, primo casu functionem habere oscillationem pro valore $x=r$, altero autem functionem habere oscillationem per intervallum a $x=a$ usque ad $x=b$ extensum, tertio denique, functionem habere oscillationes et pro singulis valoribus et per spatia quaedam finita variabilis $x$. Atque oscillationum prorsus diversa esse genera bina perspicimus.

Alque omnibus rebus, quae ad judicium de convergentia seriei (1.) ferendum spectare viderentur perpensis, adminiculo opus esse apparebat, quod in his disquisitionibus locum theorematis I. teneret, majorem vero amplecteretur generalitatem. Itaque, quod proponimus, est hocce

Theorema II. Quantitatibus $g$ et $h$ explentibus conditiones signis $0 \leqq g<h \leqq \frac{\pi}{2}$ denotatas sit functionis $f(\beta)$ indoles ea, ut per intervallum a $\beta=g$ usque ad $\beta=h$ extensum valorem teneat inter valores constantis alicujus tum positive quum negative acceptae positum, ut differentia $f(g+\delta)-f(g)$ decrescente quantitate positiva $\delta$ quantumvis exigua evadat, ut differentia $f^{\prime}(\beta+\delta)-f(\beta)$ pro $g<\beta<h$ decrescente quantitate positiva $\delta$ valorem assequatur absolute minorem potestate quadam positiva quantitatis $\delta$ in aliam constantem ducta; tum integrale $\int_{g}^{\circ h} f(\beta) \frac{\sin k \beta}{\sin \beta} d \beta$ quantitate positiva $k$ omnem modum excedente certo cuidam valori appropinquat. Qui valor, si quantitas $g$ est positiva, cifrae aequalis, si quantitas $g$ est cifrae aequalis, $=\frac{\pi}{2} f(0)$ prodit.

Cujus propositionis haec adornari potest

Demonstratio. Facillime e commentationibus illụstrissimi LejeuneDirichlet supra commemoratis perspicitur, propositionem nostram veram esse totam, dummodo valeat pars ea, quae ad hypothesin $g=0$ referat, et valeat quidem functione $f(\beta)$ per intervallum a $\beta=0$ usque ad $\beta=h$ extensum semper manente positiva. Qua de re id solum ostendemus, si functio $f(\beta)$ a $\beta=0$ usque ad $\beta=h$ conditionibus $0<f(\beta)<A$ sufficiat, si differentia $f(\delta)-f(0)$ decrescente quantitate positiva $\delta$ et ipsa infinite decrescat, si differentia $f(\beta+\delta)-f(\beta)$ pro $0<\beta<h$ decresente quantitate positiva $\delta$ valorem nanciscatur absolute minorem valore expressionis $B \delta^{\mathfrak{a}}$, ubi signis $A, B ; \mathfrak{a}$, quantitates quaedam positivae notantur, tum integralis

$$
\text { (3.) } \quad S=\int_{0}^{h} f(\beta) \frac{\sin k \beta}{\sin \beta} d \beta
$$


proprietatem esse eam, ut in infinitum crescente quantitate positiva $k$ in valorem $\frac{\pi}{2} f(0)$ convergat. Utemur autem significationibus, quibus in commentatione, quae invenitur in repertorio a cl. Moser et Dove edito, illustrissimus LejeuneDirichlet usus est, atque formulas quasdam inde depromemus. Quo loco quum quantitas $k$ numero impari $2 n+1$ aequalis ponatur (neque enim theorematis II. quidquam interest, et ad posteriorem usum sufficit, restrictionem hanc adhibere) simplicissima via reperitur valor integralis

$$
\text { (4.) } \int_{0}^{\frac{\pi}{2}} \frac{\sin k \beta}{\sin \beta} d \beta=\frac{\pi}{2} \text {. }
$$

Tum si maximum quantitatis $\frac{\pi}{k}$ multiplum, quod quantitate $h$ continetur, vocatur $\frac{r \pi}{k}$, integrale $S$ discerpitur in $(r+1)$ integralia limitibus 0 et $\frac{\pi}{k}, \frac{\pi}{k}$ et $\frac{2 \pi}{k}, \ldots$ $\frac{r \pi}{k}$ et $h$ inclusa, atque integrale (4.) in integralium ejusmodi limitibus inclusorum certum numerum, ut eveniat

$$
\begin{aligned}
& \text { (5.) } \quad \boldsymbol{S}=\boldsymbol{R}_{1}-\boldsymbol{R}_{2}+\cdots+(-1)^{r} \boldsymbol{R}_{r+1}, \\
& \text { (6.) } \quad \boldsymbol{R}_{\nu}=(-1)^{\nu-1} \int_{\frac{(\nu-1) \pi}{k}}^{\frac{v \pi}{k}} f(\beta) \frac{\sin k \beta}{\sin \beta} d \beta, \\
& \text { (7.) } \quad \frac{\pi}{2}=\varrho_{1}-\varrho_{2}+\varrho_{3}-\varrho_{4} \pm \cdots, \\
& \text { (8.) } \quad \varrho_{\nu}=(-1)^{\nu-1} \int_{\frac{(\nu-1) \pi}{k}}^{\frac{v \pi}{k}} \frac{\sin k \beta}{\sin \beta} d \beta .
\end{aligned}
$$

Dein quantitas positiva $\varrho_{\nu}$ limitibus includitur hisce

$$
\text { (9.) } \frac{2}{k} \frac{1}{\sin \frac{\nu \pi}{k}}<\varrho_{\nu}<\frac{2}{k} \frac{1}{\sin \frac{(\nu-1) \pi}{k}},
$$

et si $2 m$ numerum aliquem parem sub quantitate $r$ positum significat, fit

$$
\text { (10.) }\left\{\begin{array}{l}
\frac{\pi}{2}>\varrho_{1}-\varrho_{2}+\cdots+\varrho_{2 m-1}-\varrho_{2 m}, \\
\frac{\pi}{2}<\varrho_{1}-\varrho_{2}+\cdots+\varrho_{2 m-1}-\varrho_{2 m}+\varrho_{2 m+1},
\end{array}\right.
$$

unde pro quantitate $\varrho_{1}$ sequitur

$$
\left(10^{a} .\right) \quad \varrho_{1}<\frac{\pi}{2}+\frac{2}{k} \frac{1}{\sin \frac{\pi}{k}} .
$$


Disquisitionum nostrarum etiam interest animum advertere ad aggregatum ex partibus positivis conflatum

$$
\rho_{\nu+1}+\rho_{\nu+2}+\cdots+\rho_{\nu^{\prime}},
$$

ubi $\nu \geqq 1, \nu^{\prime} \leqq r$, atque reperire quantitatem, qua aggregati valor contineatur. Quum in expressione (8.) quantitatis $\varrho_{\nu}$ factor $\sin k \beta$ per integrationis intervallum signum non mutet neque unitatis valorem umquam excedat, habes $\varrho_{\nu}<\int_{\frac{(\nu-1) \pi}{k}}^{\frac{\nu \pi}{k}} \frac{d \beta}{\sin \beta}$ ideoque, quae hic desiderabatur, determinationem

(11.)

$$
\varrho_{\nu+1}+\varrho_{\nu+2}+\cdots+\varrho_{\nu^{\prime}}<\int_{\frac{\nu \pi}{k}}^{\frac{\nu^{\prime} \pi}{k}} \frac{d \beta}{\sin \beta}=\log \operatorname{tg} \frac{\nu^{\prime} \pi}{2 k}-\log \operatorname{tg} \frac{\nu \pi}{2 k} .
$$

Jam ponamus

$$
\left\{\begin{array}{l}
\boldsymbol{S}^{\prime}=\boldsymbol{R}_{1}-\boldsymbol{R}_{2}+\cdots+\boldsymbol{R}_{2 m-1}-\boldsymbol{R}_{2 m}, \\
\boldsymbol{S}^{\prime \prime}=\boldsymbol{R}_{2 m+1}-\boldsymbol{R}_{2 m+2}+\cdots+(-1)^{r} \boldsymbol{R}_{r+1}
\end{array}\right.
$$

ut fiat

$$
\text { (13.) } \quad \boldsymbol{S}=\boldsymbol{S}^{\prime}+\boldsymbol{S}^{\prime \prime},
$$

et videamus, quomodo se habeant aggregata $S^{\prime}$ et $S^{\prime \prime}$ ultra ullum modum crescente numero $k$. Quem ad finem in formam paullo diversam est redigenda expressio $\boldsymbol{R}_{2 t+1}-\boldsymbol{R}_{2 t+2}$, quam, si $t<m$, aggregati $\boldsymbol{S}^{\prime}$, si $t \overline{\rangle}$, aggregati $\boldsymbol{S}^{\prime \prime}$ partem esse apparet. Introducta in expressionem $\boldsymbol{R}_{v}$ nova variabili quantitate $\gamma=k \beta$ fit

$$
\boldsymbol{R}_{\nu}=(-1)^{\nu-1} \int_{(\nu-1) \pi}^{\nu \pi} f\left(\frac{\gamma}{k}\right) \frac{\sin \gamma}{\sin \frac{\gamma}{k}} \frac{d \gamma}{k}=\int_{0}^{\pi} f\left(\frac{(\nu-1) \pi+\gamma}{k}\right) \frac{\sin \gamma}{\sin \left(\frac{(\nu-1) \pi+\gamma}{k}\right)} \frac{d \gamma}{k}
$$

itaque

vel potius

$$
\text { (15.) } \boldsymbol{R}_{2 t+1}-\boldsymbol{R}_{2 t+2}=\int_{1}^{\pi}\left(\frac{f\left(\frac{2 t \pi+\gamma}{k}\right)}{\sin \frac{2 t \pi+\gamma}{k}}-\frac{f\left(\frac{(2 t+1) \pi+\gamma}{k}\right)}{\sin \frac{(2 t+1) \pi+\gamma}{k}}\right) \frac{\sin \gamma d \gamma}{k}
$$

$$
\left\{\begin{array}{c}
\boldsymbol{R}_{2 t+1}-\boldsymbol{R}_{2 t+2} \\
=\frac{1}{2} \int_{0}^{n}\left(f\left(\frac{2 t \pi+\gamma}{k}\right)+f\left(\frac{(2 t+1) \pi+\gamma}{k}\right)\right)\left(\frac{1}{\sin \frac{2 t \pi+\gamma}{k}}-\frac{1}{\sin \frac{(2 t+1) \pi+\gamma}{k}}\right) \frac{\sin \gamma d \gamma}{k} \\
+\frac{1}{2} \int_{0}^{\pi}\left(f\left(\frac{2 t \pi+\gamma}{k}\right)-f\left(\frac{(2 t+1) \pi+\gamma}{k}\right)\right)\left(\frac{1}{\sin \frac{2 t \pi+\gamma}{k}}+\frac{1}{\sin \frac{(2 t+1) \pi+\gamma}{k}}\right) \frac{\sin \gamma d \gamma}{k}
\end{array}\right.
$$


Quorum duorum integralium quoniam factores

$$
\frac{1}{\sin \frac{2 t \pi+\gamma}{k}}-\frac{1}{\sin \frac{(2 t+1) \pi+\gamma}{k}} \text { et } \sin \gamma \text { et } \frac{1}{\sin \frac{2 t \pi+\gamma}{k}}+\frac{1}{\sin \frac{(2 t+1) \pi+\gamma}{k}}
$$

per cursum integrationis positivi manent, integralis prioris valorem, dum loco factoris $f\left(\frac{2 t \pi+\gamma}{k}\right)+f\left(\frac{(2 t+1) \pi+\gamma}{k}\right)$ quantitatem aut majorem aut minorem, atque alterius integralis valorem, dum loco factoris $f\left(\frac{2 t \pi+\gamma}{k}\right)-f\left(\frac{(2 t+1) \pi+\gamma}{k}\right)$ quantitatem aut majorem aut minorem ponas, aestimare poteris. Propositum vero alio modo, si $t<m$, alio modo, si $t \overline{\overline{>}} m$ est, consequi oportet.

Priore enim casu sub signis integrationis formula (16.) adhibitis inveniuntur valores functionis $f(\beta)$ tantum ii, qui valoribus $\beta$ inter $\beta=0$ et $\beta=\frac{2 m \pi}{k}$ positis competunt. Quorum valorum secundum hypothesin positivorum et quantitate $A$ superatorum si absolute maximum $f(0)+\lambda$, absolute minimum $f(0)-\mu$ nuncupamus, summam $\frac{1}{2} f\left(\frac{2 t \pi+\gamma}{k}\right)+\frac{1}{2} f\left(\frac{(2 t+1) \pi+\gamma}{k}\right)$ inter valores $f(0)-\mu$ et $f(0)+\lambda$, differentiam $\frac{1}{2} f\left(\frac{2 t \pi+\gamma}{k}\right)-\frac{1}{2} f\left(\frac{(2 t+1) \pi+\gamma}{k}\right)$ inter valores $\pm\left(\frac{\lambda+\mu}{2}\right)$ contineri elucet, unde prodeunt relationes

$$
\left\{\begin{aligned}
t<m ; & \boldsymbol{R}_{2 t+1}-\boldsymbol{R}_{2 t+2}<(f(\mathbf{0})+\lambda)\left(\varrho_{2 t+1}-\varrho_{2 t+2}\right)+\frac{\lambda+\mu}{2}\left(\varrho_{2 t+1}+\varrho_{2 t+2}\right), \\
& \boldsymbol{R}_{2 t+1}-\boldsymbol{R}_{2 t+2}>(f(0)-\mu)\left(\boldsymbol{\varrho}_{2 t+1}-\varrho_{2 t+2}\right)-\frac{\lambda+\mu}{2}\left(\varrho_{2 t+1}+\varrho_{2 t+2}\right) .
\end{aligned}\right.
$$

Altero autem casu, ubi $t \geqq m$ assumitur, sub signis integrationis formula (16.) usitatis inveniuntur valores functionis $f(\beta)$ tantum ii. qui valoribus $\beta$ inter $\beta=\frac{2 m \pi}{k}$ et $\beta=h$ positis respondent. Qua in re monemus summam $\frac{1}{2} f\left(\frac{2 t \pi+\gamma}{k}\right)+\frac{1}{2} f\left(\frac{(2 t+1) \pi+\gamma)}{k}\right)$ esse positivam et quantitate $A$ minorem. In aestimanda vero differentia $\frac{1}{2} f\left(\frac{2 t \pi+\gamma}{k}\right)-\frac{1}{2} f\left(\frac{(2 t+1) \pi+\gamma}{k}\right)$, ubi $\gamma$ valores a $\gamma=0$ usque ad $\gamma=\pi$ omnes percurrit, numerum $k$ tam magnum fingere licet, ut iis, quae de differentia $f(\beta+\delta)-f(\delta)$ posita sint, uti queamus. Quia enim valor absolutus differentiae $f(\beta+\delta)-f(\delta)$, si $0<\beta<h$, decrescente quantitate positiva $\delta$ infra quantitatem $B \delta^{a}$ deprimitur et nostro casu $\frac{2 t \pi+\gamma}{k} \geqq \frac{2 m \pi}{k}$, 
$\delta=\frac{\pi}{k}$ est, differentiam $\frac{1}{2} f\left(\frac{2 t \pi+\gamma}{k}\right)-\frac{1}{2} f\left(\frac{(2 t+1) \pi+\gamma}{k}\right)$ limitibus $\pm \frac{1}{2} B\left(\frac{\pi}{k}\right)^{a}$ inclusam esse apparet. Atque nanciscimur relationes hasce

$$
\begin{aligned}
& \boldsymbol{R}_{2 t+1}-\boldsymbol{R}_{2 t+2}<A\left(\varrho_{2 t+1}-\varrho_{2 t+2}\right)+\frac{1}{2} B\left(\frac{\pi}{k}\right)^{\mathfrak{a}}\left(\varrho_{2 t+1}+\varrho_{2 t+2}\right), \\
& \boldsymbol{R}_{2 t+1}-\boldsymbol{R}_{2 t+2}>-\frac{1}{2} B\left(\frac{\pi}{k}\right)^{\mathfrak{a}}\left(\varrho_{2 t+1}+\varrho_{2 t+2}\right) .
\end{aligned}
$$

Formulae nostrae (17.) et (18.) idoneae sunt, ut limitibus circumdentur aggregatorum $S^{\prime}$ et $S^{\prime \prime}$ partes omnes, si $r$ numero pari $2 q$ aequalis est, excepta quantitate $R_{r+1}$, si $r$ numero impari $2 q+1$ aequalis est, excepta expressione $\boldsymbol{R}_{r}-\boldsymbol{R}_{r+1}$. Quum autem ex iis, quae posita sunt, sequatur $\boldsymbol{R}_{r}>\mathbf{0}, \boldsymbol{R}_{r+1}>\mathbf{0}$, $\boldsymbol{R}_{r}<A \varrho_{r}, \boldsymbol{R}_{r+1}<A \varrho_{r+1}, \varrho_{r+1}<\varrho_{r}$ dubium esse non potest, quin quantitas $R_{r+1}$, si $r=2 q$, quantitas $R_{r}-R_{r+1}$, si $r=2 q+1$, iisdem limitibus $\pm A \varrho_{2 q}$ contineatur. Itaque hunc in modum aestimare licet aggregata $S^{\prime}$ et $S^{\prime \prime}$

(19.)

$$
\left\{\begin{array}{l}
S^{\prime}<(f(0)+\lambda)\left(\varrho_{1}-\varrho_{2}+\cdots+\varrho_{2 m-1}-\varrho_{2 m}\right)+\frac{\lambda+\mu}{2}\left(\varrho_{1}+\varrho_{2}+\cdots+\varrho_{2 m-1}+\varrho_{2 m}\right), \\
S^{\prime}>(f(0)-\mu)\left(\varrho_{1}-\varrho_{2}+\cdots+\varrho_{2 m-1}-\varrho_{2 m}\right)-\frac{\lambda+\mu}{2}\left(\varrho_{1}+\varrho_{2}+\cdots+\varrho_{2 m-1}+\varrho_{2 m}\right), \\
S^{\prime \prime}<A\left(\varrho_{2 m+1}-\varrho_{2 m+2}+\cdots+\varrho_{2 q-1}-\varrho_{2 q}\right)+\frac{1}{2} B\left(\frac{\pi}{k}\right)^{a}\left(\varrho_{2 m+1}+\cdots+\varrho_{2 q}\right)+A \varrho_{2 q}, \\
S^{\prime \prime}>-\frac{1}{2} B\left(\frac{\pi}{k}\right)^{a}\left(\varrho_{2 m+1}+\cdots+\varrho_{2 q}\right)-A \varrho_{2 q} .
\end{array}\right.
$$

Tum ex formulis (9.), (10.), $\left(10^{a}.\right),(11$.$) derivantur sequentes$

$$
\left\{\begin{array}{l}
\frac{\pi}{2}-\frac{2}{k} \frac{1}{\sin \frac{2 m \pi}{k}}<\varrho_{1}-\varrho_{2}+\cdots+\varrho_{2 m-1}-\varrho_{2 m}<\frac{\pi}{2}, \\
\varrho_{2 m+1}-\varrho_{2 m+2}+\cdots+\varrho_{2 q-1}-\varrho_{2 q}<\frac{2}{k} \frac{1}{\sin \frac{2 m \pi}{k}}, \quad \varrho_{2 q}<\frac{2}{k} \frac{1}{\sin \frac{(2 q-1) \pi}{k}} \\
\varrho_{1}+\varrho_{2}+\cdots+\varrho_{2 m-1}+\varrho_{2 m}<\frac{\pi}{2}+\frac{2}{k} \frac{1}{\sin \frac{\pi}{k}}+\log \operatorname{tg} \frac{m \pi}{k}-\log \operatorname{tg} \frac{\pi}{2 k}, \\
\varrho_{2 m+1}+\cdots+\varrho_{2 q}<\log \operatorname{tg} \frac{q \pi}{k}-\log \operatorname{tg} \frac{m \pi}{k},
\end{array}\right.
$$

quibus in (19.) substitutis prodeunt novae

Journal für Mathematik Bd. LXIII. Heft 4. 
(21.)

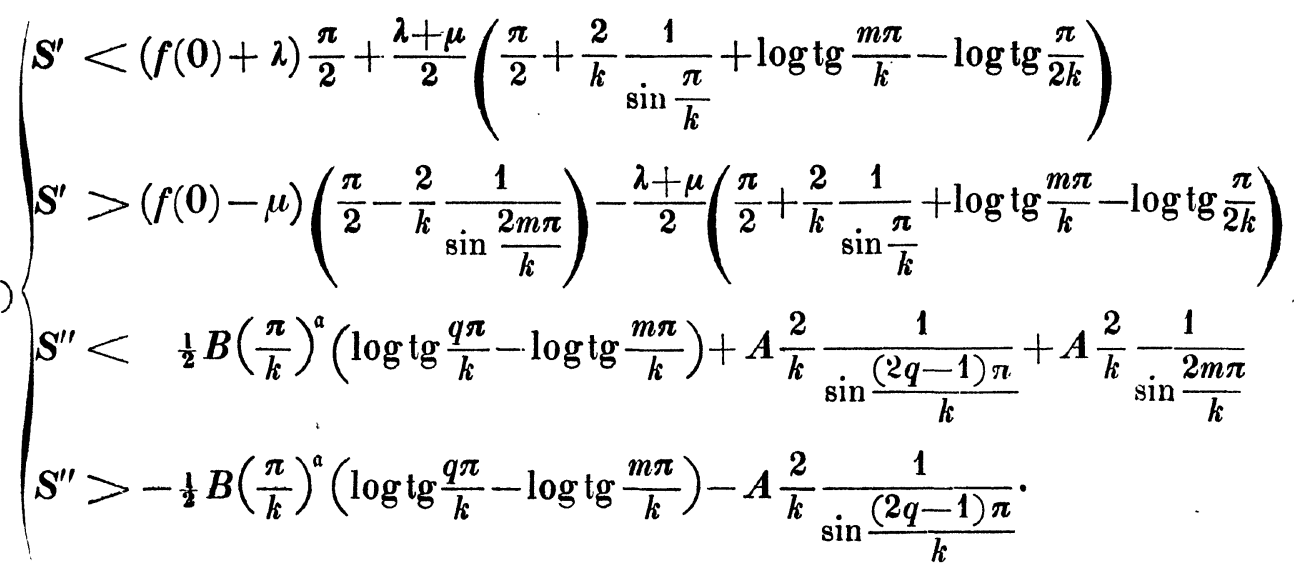

Quibus relationibus ut concludatur propositionem nostram esse veram, numerum $m$ certum quendam retinere valorem, dum numerus $k$ continuo crescit, ponimus. Quum autem per hypothesin differentia $f(\delta)-f(0)$ decrescente $\delta$ quamvis exigua fiat, quum $f(0)+\lambda$ absolute maximus, $f(0)-\mu$ absolute minimus sit valor functionis $f(\beta)$ variabili $\beta$ spatium a $\beta=0$ usque ad $\beta=\frac{2 m \pi}{k}$ percurrente, et quum quantitas $\frac{2 m \pi}{k}$ continuo decrescat, quantitates $\lambda$ et $u$ facere non possunt, quin et ipsae infinite decrescant. - Tum ex notis analyseos principiis sequitur

$$
\left\{\begin{aligned}
\frac{2}{k} \frac{1}{\sin \frac{\pi}{k}+\log \operatorname{tg} \frac{m \pi}{k}-\log \operatorname{tg} \frac{\pi}{2 k}} & =\frac{2}{\pi}+\log 2 m+w \\
\frac{2}{k} \frac{1}{\sin \frac{2 m \pi}{k}} & =\frac{1}{m \pi}+w^{\prime} \\
\log \operatorname{tg} \frac{q \pi}{k}-\log \operatorname{tg} \frac{m \pi}{k} & =\log \operatorname{tg} \frac{h}{2}-\log \frac{m \pi}{k}+w^{\prime \prime} \\
\frac{2}{k} \frac{1}{\sin \frac{(2 q-1) \pi}{k}} & =\frac{2}{k} \frac{1}{\sin h}+w^{\prime \prime \prime},
\end{aligned}\right.
$$

ubi signis $w, w^{\prime}, w^{\prime \prime}, w^{\prime \prime \prime}$ significantur quantitates, in infinitum crescente numero $k$, ad cifram quam proxime accedentes. Quibus de causis relationes (21.) in hanc redigere licet formam 


$$
\left\{\begin{array}{l}
S^{\prime}<(f(0)+\lambda) \frac{\pi}{2}+\frac{\lambda+\mu}{2}\left(\frac{\pi}{2}+\frac{2}{\pi}+\log 2 m+w\right) \\
S^{\prime}>(f(0)-\mu)\left(\frac{\pi}{2}-\frac{1}{m \pi}-w^{\prime}\right)-\frac{\lambda+\mu}{2}\left(\frac{\pi}{2}+\frac{2}{\pi}+\log 2 m+w\right) \\
S^{\prime \prime}<\frac{1}{2} B\left(\frac{\pi}{k}\right)^{a}\left(\log \operatorname{tg} \frac{h}{2}-\log \frac{m \pi}{k}+w^{\prime \prime}\right)+A\left(\frac{2}{k} \frac{1}{\sin h}+w^{\prime \prime \prime}+\frac{1}{m \pi}+w^{\prime}\right) \\
S^{\prime \prime}>-\frac{1}{2} B\left(\frac{\pi}{k}\right)^{a}\left(\log \operatorname{tg} \frac{h}{2}-\log \frac{m \pi}{k}+w^{\prime \prime}\right)-A\left(\frac{2}{k} \frac{1}{\sin h}+w^{\prime \prime \prime}\right) .
\end{array}\right.
$$

Quibus in formulis id intuemur, expressionis

$$
\frac{1}{2} B\left(\frac{\pi}{k}\right)^{a}\left(\log \operatorname{tg} \frac{h}{2}-\log \frac{m \pi}{k}\right)
$$

quae expressioni $\frac{1}{2} B\left(\frac{\pi}{k}\right)^{a}\left(\log \operatorname{tg} \frac{h}{2}-\log m\right)+\frac{1}{2} B\left(\frac{\pi}{k}\right)^{a} \log \left(\frac{k}{\pi}\right)$ aequalis est, valorem crescente numero $k$ minorem fieri quavis quantitate data, quia aggregatum $\log \operatorname{tg} \frac{h}{2}-\log m$ certum tenet valorem, potestas vero $\left(\frac{\pi}{k}\right)^{a}$ decrescit, functio denique $\left(\frac{\pi}{k}\right)^{a} \log \left(\frac{k}{\pi}\right)$, quamvis augeatur factor $\log \left(\frac{k}{\pi}\right)$, et ipsa decrescit pro omni valore a positivo. Atque hac in re sunt nervi demonstrationis nostrae. Si itaque quantitates quaedam, quae crescente numero $k$ ad cifram appropinquent, nuncupantur $\eta, \eta^{\prime}, \eta^{\prime \prime}, \eta^{\prime \prime \prime}$, consequimur formulas

$$
\begin{cases}S^{\prime}<\frac{\pi}{2} f(0)+\eta, & S^{\prime}>\frac{\pi}{2} f(0)-\frac{A}{m \pi}+\eta^{\prime}, \\ S^{\prime \prime}<\frac{A}{m \pi}+\eta^{\prime \prime}, & S^{\prime \prime}>-\eta^{\prime \prime \prime} .\end{cases}
$$

Unde apparet, dummodo numerus $m$ ita assumatur, ut quantitas $\frac{A}{m \pi}$ minor fiat exigua quadam quantitate ad libitum data, id quod semper fieri posse res ipsa indicat, aggregatum $S^{\prime}$ includi limitibus, quae quam minimum velis a valore $\frac{\pi}{2} f(0)$ differant, aggregatum $S^{\prime \prime}$ autem includi limitibus, quae quam minimum velis a valore cifrae distent. Quum igitur continuo crescente numero $k$ aggregatum $S^{\prime}$ ad quantitatem $\frac{\pi}{2} f(0)$, aggregatum $S^{\prime \prime}$ ad cifram quam proxime accedat, dubitari non potest, quin aggregatum $S=S^{\prime}+S^{\prime \prime}$ convergat in valorem $\frac{\pi}{2} f(0)$. Q. E. D.

Accurata demonstratione munito theoremate II. aditus patet ad indolem seriei (1.) casu nostro tertio melius cognoscendam. Est enim convergens et valori $\frac{1}{2}(\varphi(x-\varepsilon)+\varphi(x+\varepsilon))$, denotante $\varepsilon$ quantitatem infinite parvam, quicunque valor inter $-\pi$ et $+\pi$ situs quantitati $x$ conferatur, aequalis semper, si functio 
$\varphi(x)$ pro singulis tantum variabilis valoribus praebet oscillationes, et semper una tantum hypothesi excepta, si functio $\varphi(x)$ per finitas intervalli partes habet oscillationes. Quae hypothesis est ea, ut per finitam quandam intervalli partem differentia $\varphi(x+\delta)-\varphi(x)$, quam decrescente quantitate $\delta$ et ipsam ad cifram quam proxime accedere ex continuitate functionis $\varphi(x)$ per eam intervalli partem servanda sequitur (et pro continuitatis definitione habendum est *)), ita tamen minuatur, ut pro omnibus variabilis valoribus spatio illo contentis nulla quantitatis $\delta$ fingi possit potestas positiva, quam non superet. Atque hoc evenire apparet, si differentia $\varphi(x+\delta)-\varphi(x)$ tali modo vel etiam lentius, quam functio $\frac{1}{\log \frac{1}{\delta}}$ decrescit**). Neque vero demonstrationis vis deletur, si in spatiis, per quae functio $\varphi(x)$ oscillationem habet, pro singulis variabilis $x$ valoribus accidit, ut differentia $\varphi(x+\delta)-\varphi(x)$ modo dicto se habeat.

Hypothesi illa excepta semper fieri potest, ut intervallum functionis $\varphi(x)$ a $-\pi$ usque ad $+\pi$ extensum in finitum numerum spatiorum dirimatur, quorum limites invenies, dum secundum magnitudinem ordinaveris valores variabilis $x$ eos, $1^{\prime \prime}$ quibus in functione $\varphi(x)$ continuitas deleatur, $2^{\prime \prime}$ quibus in functione $\varphi(x)$ valores maximi et minimi, ut ita dicam, singuli competant, $3^{0}$ quibus singulis functio $\varphi(x)$ praebeat oscillationes, $4^{\prime \prime}$ qui sint limites spatiorum finitorum, per quae functio $\varphi(x)$ habeat oscillationes, $5^{0}$ denique quibus singulis, dum in ejusmodi spatiis jacent, differentia $\varphi(x+\delta)-\varphi(\delta)$ decrescente quantitate $\delta$ lentius minuatur ulla quantitatis $\delta$ potestate positiva. Tum integrationis intervallo in integrali (2.) eundem in modum distributo integrale (2.) aggregato integralium formam eandem habentium aequale fieri, et crescente numero $k$ eorum integralium quodque ad certum quendam valorem appropinquare elucet, qui vel theoremate I. vel theoremate II. facile designetur, unde, quae proposuimus, sequuntur.

Quae autem seriei (1.), posita illa hypothesi, sit indoles, speramus fore, ut alio loco et tempore quaerere nobis liceat.

Scripsi Bonnae die X. M. Aprilis A. MDCCCLXIV.

*) Diar. Crell. Vol. IV, pag. 159.

**) Allgemeine Lehrsätze in Beziehung auf die im verkehrten Verhältnisse des Quadrats der Entfernung wirkenden Anziehungs- und Abstossungskrätte. Art. 16. auct. Gauss. 